\title{
Correlation of prognostic factors with MRI findings in malignant breast lesions
}

\author{
Kerim Temiz ${ }^{1 *}$ D, Pelin Seher Oztekin ${ }^{1}$, Sema Hucumenoglu ${ }^{2}$, Enver Necip Koseoglu ${ }^{1}$ and Pinar Nercis Kosar ${ }^{1}$
}

\begin{abstract}
Background: Magnetic resonance imaging (MRI) of the breast represents the most sensitive imaging modality in the detection of breast cancer, with a reported sensitivity between 94 and 100\%. We aim to detect the correlation between MRI findings and pathologically detected prognostic factors in malignant breast lesions.

Breast parenchymal density distribution, background parenchymal enhancement pattern, lesion's morphologic features, T2WI signal characteristics, contrast enhancement, time/signal intensity curves, and lesions localizations in breast were evaluated using dynamic MRI images. Histopathological diagnosis, maximum measurements of the lesion, histological grade, presence of estrogen and/or progesterone receptors, c-erb B2, and Ki-67 parameters were noted as prognostic factors.

Results: We cannot detect any relationship between the breast parenchymal density and prognostic factors. Mild background breast enhancement is related with ER presence, a good prognostic factor. Histopathological grade of the lesions augmented with the increase in the lesion diameters. ADC values are not related with prognostic factors.

Conclusion: A mild background enhancement, an intermediate signal intensity on T2WI, a high tpeak value, and absence of pathological axillary lymph node are found to be related with good prognostic factors. An irregular contour, a huge diameter, having a type III kinetic curve, a high slopei value, and presence of pathological axillary lymph node are found to be related with poor prognostic factors. MRI can be used to predict prognostic factors in breast cancer cases.
\end{abstract}

Keywords: Breast, Malignancy, Prognostic factor, MRI

\section{Background}

Breast cancer, being the most frequent cancer in females, is an important problem. Mammography and ultrasonography (US) can be insufficient in evaluating lesions behaviors, multicentricity, and distinction of residue tumorgranulation tissue and after treatment follow-up. In these situations, magnetic resonance imaging (MRI) becomes an important tool in routine imaging [1-3].

MRI of the breast represents the most sensitive imaging modality in the detection of breast cancer, with a reported sensitivity between 94 and $100 \%$ [1]. In the last decades, in order to increase the specificity of breast

\footnotetext{
* Correspondence: kerimtmz@yahoo.com

'Department of Radiology, Ankara Training and Research Hospital, Ankara, Turkey

Full list of author information is available at the end of the article
}

MRI in describing lesions, diffusion-weighted images (DWI) are added into the classical conventional and dynamic MRI sequences.

Especially in the past two decades, advances in the diagnosis and treatment of breast cancer decreased the mortality rates. Prognostic factors have been gaining an increasing importance in directing the type of treatment. Recently, seeing the fact that there is an increasing rate of relapse especially in patients who do not have an axillary metastatic lymph node, researchers began to study on minor deterministic findings, called secondary prognostic factors [4].

In this study, we aim to detect the correlation between MRI findings and pathologically detected prognostic factors in malignant breast lesions. 


\section{Methods}

\section{Patient population}

This retrospective study involved a total 59 patients who had both a dynamic contrast-enhanced breast MRI and pathologically confirmed diagnosis between 30 and 76 years of age. The study protocol was approved by the Institutional Ethics Committee.

\section{Breast MRI technique and interpretation}

Lesion characteristics were classified according to Breast Imaging Reporting and Data System (BI-RADS). When a patient had more than one lesion, the biggest one was preferred for the evaluation. Dynamic contrast-enhanced and diffusion-weighted MRI were evaluated by two radiologists.

Breast MRI examinations were performed with a $1.5-\mathrm{T}$ whole-body imaging system (Signa Excite, GE Healthcare, Milwaukee, WI, USA). The patients were scanned in the prone position with the breast suspended in a four-channel breast coil. Pre-contrast transverse acquisitions were performed using a T1-weighted fast spin echo sequence and transverse T2 -weighted fast spin echo short-tau inversion recovery (STIR) imaging, pre-contrast sagittal acquisitions were performed using a T2-weighted fast spin echo sequence imaging with fat suppression. DWI were acquired with fat saturation using axial echo planar imaging (EPI) sequences at $\mathrm{b}-1000 \mathrm{~s} / \mathrm{mm}^{2}$ before contrast administration. Sagittal pre- and post-contrast dynamic imaging was performed using a 3D multi-phase fast gradient echo pulse sequence called VIBRANT. Additionally, transverse post-contrast T1-weighted images were acquired using the fast spoiled gradient-recalledecho sequence in the same manner as it was used to acquire the pre-contrast images, without a change in the patient's position. Subtraction images were created. The patients were given a bolus intravenous injection of gadolinium contrast $(0.2 \mathrm{mmol} / \mathrm{kg})$. Both morphological features and kinetic characteristics of the lesions were examined.

Breast parenchymal density distribution, background parenchymal enhancement pattern, lesion's morphologic features, T2WI signal characteristics, contrast enhancement characteristics, time/signal intensity curves, and lesions localizations in breast were evaluated using dynamic MRI images.

Contrast enhancement ratio (CER) was calculated in two different times for all lesions. For the lesions who had type II or III kinetic curve, first measurement was performed when the lesion showed maximum contrast enhancement. For type I kinetic curve lesions, first measurement was performed via first images with contrast enhancement. Second measurements were performed at the maximal phase of wash-out if the lesion has type III kinetic curve. For the lesions with a type I or
II kinetic curve; second measurement were performed by using the last images with contrast enhancement. Then, initial slope value was calculated with the following formula:

$$
\text { Slopei }=\frac{\text { CERpeak }}{\text { tpeak }}
$$

CERpeak = Maximum contrast enhancement value tpeak $=$ Time to reach maximum contrast enhancement value

Contrast enhancement ratio was calculated with following formula:

$$
\mathrm{CER}=\frac{\mathrm{SI}(\text { post })-\mathrm{SI}(\text { pre })}{\mathrm{SI}(\text { pre })} \times 100
$$

$\mathrm{CER}=$ Contrast enhancement ratio SI (pre) = Basic signal intensity

SI (post) = Signal intensity after contrast enhancement at a specific time.

Mean contrast enhancement ratio and apparent diffusion coefficient (ADC) values were measured by excluding the cystic-necrotic areas and using a ROI as large as possible. Measurements were repeated three times to obtain a mean value.

Histopathological diagnosis, maximum measurements of the lesion, histological grade, presence of estrogen and/or progesterone receptors, c-erb B2, and Ki-67 parameters were noted as histological/immunohistochemical prognostic factors.

\section{Statistical methods}

Statistical analyses were performed using IBM SPSS Statistics 21. Continuous parameters were presented as mean \pm standard deviation and skewed continuous parameters were performed by Mann-Whitney $U$ test. Kruskal-Wallis test was used to compare between more than two groups. For the different values obtained from Kruskal-Wallis test, Conover test of variances was used. Categorical data were presented as frequencies and percentages and were compared using Fisher exact or chisquare tests where appropriate.

A two-tailed $p<0.05$ was considered statistically significant. A $p$ value between 0.1 and 0.05 was considered as a weak proof for a relationship/difference between analyzed factors.

\section{Results}

Fifty-nine women, between the ages of 30 and 76 (median 51, mean 51.25) were included into the current study.

The breast parenchyma density and parenchyma enhancement pattern of the patients are shown in Table 1. 
Table 1 The breast parenchyma density and parenchymal enhancement pattern of the patients

\begin{tabular}{lllll}
\hline $\begin{array}{l}\text { Parenchymal } \\
\text { density }\end{array}$ & \multicolumn{4}{l}{ Enhancement pattern } \\
\cline { 2 - 5 } & Minimal & Mild & Moderate & Strong \\
\hline Type A & 5 & 0 & 0 & 5 \\
Type B & 18 & 0 & 0 & 18 \\
Type C & 18 & 11 & 2 & 31 \\
Type D & 2 & 1 & 2 & 5 \\
TOTAL & 43 & 12 & 4 & 59 \\
\hline
\end{tabular}

The diameters of the lesions were measured between $7 \mathrm{~mm}$ and $90 \mathrm{~mm}$ (mean 25.59, median $20 \mathrm{~mm}$ ).

Thirty-three of 59 (56\%) lesions were located at right breast, and 26/59 (44\%) were located at left breast. Four of $59(6.8 \%)$ lesions were bilateral. Forty-one of 59 (69.4\%) lesions were unifocal, 8/59 (13.6\%) lesions were multifocal, and 6/59 (10.2\%) lesions were multifocal and multicentric. Table 2 shows the distribution of pathological diagnosis of all lesions.

Fifty-one of 59 (86. 4\%) lesions were presented as enhancing masses (47 irregular shaped, 4 oval shaped; none of them was round). Four oval shaped lesions were diagnosed as $2 / 4$ invasive ductal carcinoma (IDC), $1 / 4$ tubular carcinoma (TC), and $1 / 4$ ductal carcinoma in situ (DCIS).

There were four non mass-like enhancement, diagnosed as $2 / 4$ DCIS, $1 / 4 \mathrm{TC}$, and $1 / 4$ invasive cribriform carcinoma.

Signal characteristics and kinetic curve types of the lesions are shown in Table 3.

Primary CERs of the lesions (CER1) vary between 23.11 and 245.28, mean 118.23 (median 122.27). Secondary CERs (CER2) vary between 18.58 and 191.1, mean 99.27 (median 101.29). Tpeak values changes between 50 and $250 \mathrm{~s}$, mean 89.831 (median $50 \mathrm{~s}$ ). Slopei values vary between 0.2349 and 4.9256, mean 1.7365 (median 1.4929). We had only 43 patients' DWI images and ADC maps. ADC values of these patients vary between 0.138 $\times 10^{-3}$ and $1.88 \times 10^{-3} \mathrm{~mm} / \mathrm{s}$, mean $0.846 \times 10^{-3} \mathrm{~mm} / \mathrm{s}$ (median $0.806 \times 10^{-3} \mathrm{~mm} / \mathrm{s}$ ).

Table 2 The distribution of pathological diagnosis

\begin{tabular}{llll}
\hline Pathological diagnosis & Number & Percent & Total \\
\hline Ductal carcinoma in situ & 5 & 8.5 & \\
Invasive ductal carcinoma & 43 & 72.8 & \\
Invasive lobular carcinoma & 4 & 6.8 & \\
Tubular carcinoma & 3 & 5.1 & 59 \\
Mucinous carcinoma & 2 & 3.4 & \\
Invasive cribriform carcinoma & 1 & 1.7 & \\
Neuroendocrine carcinoma & 1 & 1.7 & \\
\hline
\end{tabular}

Twenty-one of 59 (35.5\%) patients had pathologically confirmed axillary lymph nodes on MR images; 38/50 (64.5\%) patients did not have any pathological axillary lymph node.

Estrogen receptor (ER), progesterone receptor (PR), and c-erb B2 amplification characteristics of lesions are shown in Table 4. Ki-67 proliferation index was evaluated in 24 lesions; $13 / 24$ had high and 11/24 had low index.

We cannot detect any relationship between the breast parenchymal density and prognostic factors. Mild background breast enhancement is related with ER presence, a good prognostic factor.

There is a weak relationship between an irregular shape and high Ki-67 index, a poor diagnostic factor ( $p$ $=0.096$ ) (Figs. 1 and 2). A weak relationship was also detected between intermediate signal intensity on T2WIs and not-augmented c-erb B2, a good prognostic factor $(p=0.092)$. Histopathological grade of the lesions augmented with the increase in the lesion diameters. In addition, lesions' diameter has a negative correlation with absence of $\mathrm{PR}$ and ER, poor prognostic factors $(p=$ 0.056).

Type III kinetic curve is significantly related with a high Ki-67 index, a poor prognostic factor (Fig. 3). There is a weak relationship between a high tpeak value and not-augmented c-erb B2, a good prognostic factor ( $p=$ 0.053). Another week relationships detected between slopei values and ER absence, a poor diagnostic factor ( $p=$ 0.090).

ADC values are not related with prognostic factors.

Presence of axillary pathological lymph nodes is related with a high Ki-67 index and high histopathological grade. There is weak relationship between axillary pathological lymph node presence and early diagnosis age, a poor diagnostic factor $(p=0.055)$.

Absence of axillary pathological lymph nodes is related with good prognostic factors, presence of ER and PR.

\section{Discussion}

Dynamic breast MRI became an important tool for diagnosing and grading breast cancer. With the help of contrast medium not only morphology of the cancer but also its functional characteristics can be showed [5]. The potential relationship between MRI characteristics and prognostic factors is discussed by some researchers; however, there are controversial results because of different MRI techniques and evaluating criteria [6].

According to current data a mild background breast enhancement is related with ER presence, a good prognostic marker. Also, another current study stated that mild background parenchymal enhancement predicts good prognosis, and it is related with a negative HER2 expression. This condition might be the consequence of 
Table 3 Signal characteristics and kinetic curve types of the lesions

\begin{tabular}{|c|c|c|c|c|c|c|c|c|}
\hline \multicolumn{3}{|l|}{ T2WI sıgnal } & \multicolumn{3}{|c|}{ Contrast-enhanced images } & \multicolumn{3}{|c|}{ Kınetıc curve type } \\
\hline Hypointense & Intermediate & Hypointense & Homogeneous & Heterogeneous & Peripheral & Type 1 & Type 2 & Type 3 \\
\hline $11,18.6 \%$ & $40,67.8 \%$ & $8,13.6 \%$ & $15,25.4 \%$ & $34,57.6 \%$ & $10,17 \%$ & $4,6.8 \%$ & $7,11.9 \%$ & $48,81.3 \%$ \\
\hline
\end{tabular}

rarity of angiogenic-inflammatory factors in less aggressive ER-positive lesions [7, 8]. In line with the literature, we defined a positive correlation with mild parenchymal enhancement and ER presence/good prognosis.

There are controversial studies about lesions' shape and prognostic factors especially histological grade. Shapes of the lesions on MRI were stated to be related with histopathological grade and Ki-67 values in one study; however, this relationship cannot be confirmed by other studies $[6,9,10]$. According to our results, irregular lesion shape is weakly related with a high Ki-67 index, but there is no relationship between lesion shape and histological grade.

T2* values were considered to be an imaging biomarker related with prognosis in breast, prostate, and head/neck cancer [11]. Partially similar with the literature, we found that intermediate signal intensity on T2WI was correlated with non-augmented c-erb B2 status/good prognosis.

Peripherally contrast enhancement is described as a poor prognostic factor in the literature, it is found to be related with ER absence, high histological grade, and high Ki-67 index $[6,12]$. Also, a high contrast enhancement ratio was found to be related with a higher Ki-67 index and a worse prognosis [13]. However, there is an another study saying contrast enhancement pattern is not related with prognostic factors [14]. In our study, we cannot detect any relationship between contrast enhancement pattern and prognostic factors.

We found a relationship between a type III kinetic curve and a high Ki-67 index, consistent with the literature $[6,12,14,15]$. We cannot detect any relationship

Table 4 Estrogen receptor (ER), progesterone receptor (PR), and c-erb B2 amplification characteristics of the lesions

\begin{tabular}{|c|c|c|c|c|c|c|}
\hline \multirow[t]{2}{*}{ Pathological diagnosis } & \multicolumn{2}{|l|}{ ER } & \multicolumn{2}{|l|}{$\mathrm{PR}$} & \multicolumn{2}{|c|}{ c-erb B2 } \\
\hline & + & - & + & - & + & - \\
\hline Ductal carcinoma in situ & 5 & 0 & 4 & 1 & 3 & 2 \\
\hline Invasive ductal carcinoma & 33 & 10 & 23 & 20 & 24 & 19 \\
\hline Invasive lobular carcinoma & 4 & 0 & 3 & 1 & 1 & 3 \\
\hline Tubular carcinoma & 2 & 1 & 2 & 1 & 2 & 1 \\
\hline Mucinous carcinoma & 2 & 0 & 1 & 1 & 1 & 1 \\
\hline Invasive cribriform carcinoma & 1 & 0 & 1 & 0 & 1 & 0 \\
\hline Neuroendocrine carcinoma & 0 & 1 & 0 & 1 & 0 & 1 \\
\hline Total & 47 & 12 & 34 & 25 & 32 & 27 \\
\hline
\end{tabular}

between kinetic curve type and histopathological grade, ER presence/absence, similar to the literature $[15,16]$. Contrarily to the literature, we found that kinetic curve is not related with c-erb B2 levels [16]. This situation can be explained by the minority of our population with a c-erb B2 data.

We cannot show any relationship between lesions' diameters and kinetic curves. We also showed that a greater diameter is related with PR and ER absence. Our results are consistent with the literature [17]. Also, in the literature, it is stated that an augmented c-erb B2 causes the lesions to be greater in diameter, but we cannot show such a relationship [16].

In English literature, most of the studies found no relationship between CER and prognostic factors [18-22]; on the other hand, a more recent study defined a significant relationship with a high CER and high Ki-67 index [13]. In our study, we cannot demonstrate any relationship between CER and prognostic factors, in line with most of the previous studies.

Some studies showed that a high CER is related with the presence of an axillary pathological lymph node [19, 23], but we cannot find such a relationship in our study.

A low tpeak value is found to be related with some poor prognostic factors such as high histopathological grade, an augmented c-erb B2, ER absence, and a high Ki-67 index [6, 12, 24]. Also, maximum enhancement was found to be related with higher Ki-67 index [25]. We found a weak correlation between a low tpeak value and an augmented c-erb B2, but no relationship was found for other prognostic factors.

There are studies indicating a positive correlation between slopei value and histopathological grade, Ki-67 index $[12,25]$. We cannot confirm these relationships in our study.

We found a relationship between the presence of axillary pathological lymph node and bigger lesion diameter, high histopathological grade, and high Ki-67 index, consistent with the literature $[26,27]$. Also, absence of axillary pathological lymph node is found to be related with ER and PR presence, consistent with the literature [28, 29]. A weak relationship between axillary pathological lymph node presence and young patient age was detected. This is consistent with the readily known fact that early beginning breast cancers have poor prognosis [30].

Low ADC values were found to be related with the presence of axillary pathological lymph node in some studies, but there are also contradictory results [31, 32]. 


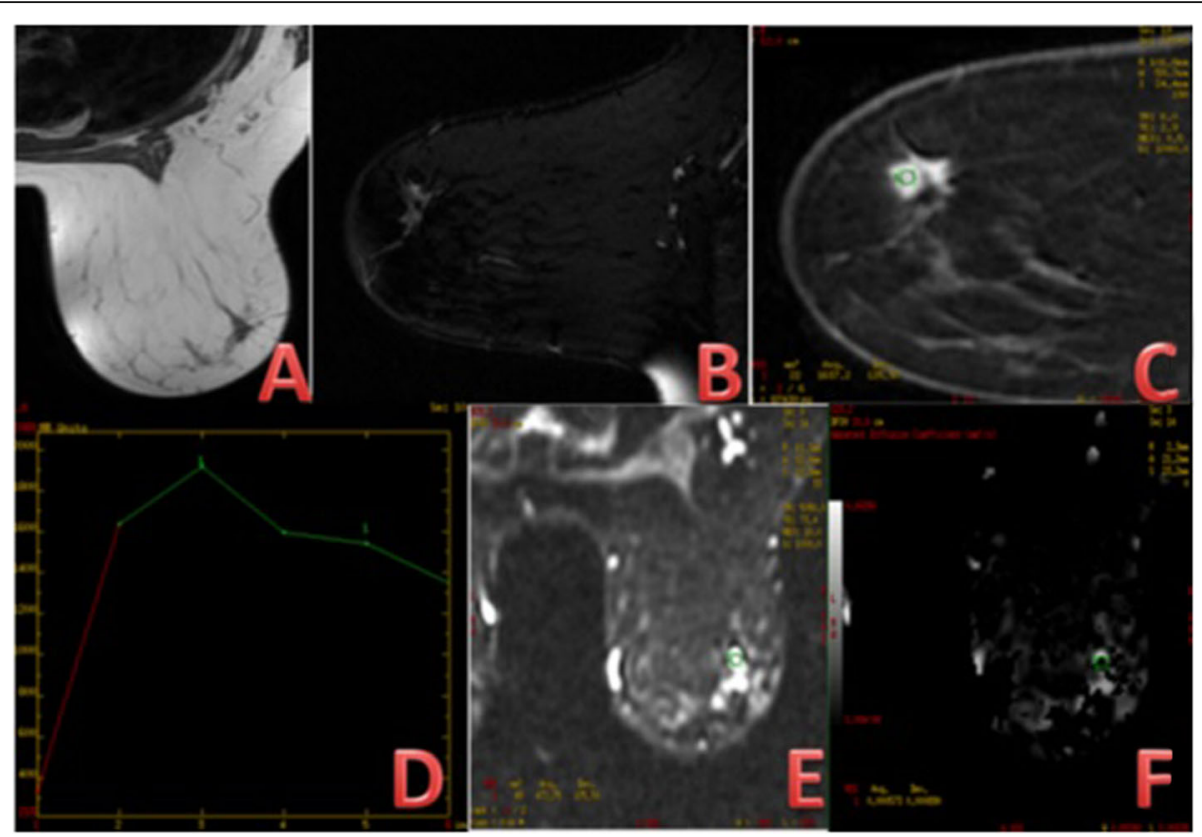

Fig. 1 52-year-old women, grade I invasive ductal carcinoma, ER (+), PR (-) c-erb B2 (+), high Ki-67 index, and no axillary pathological lymph node detected on MRI. Breast density is classified as type a on T1WI axial image (a). An irregular shaped lesion, measured $15 \mathrm{~mm}$ in diameter, is seen on sagittal fat-saturated T2WI (b) and sagittal fat-saturated, post-contrast T1WI (c). Kinetic curve of the lesion is type 3 and $t_{\text {peak }}$ value is $100 \mathrm{~s}$ (d). The lesion is hyperintense on DWI (e) and hypointense on ADC map (f)

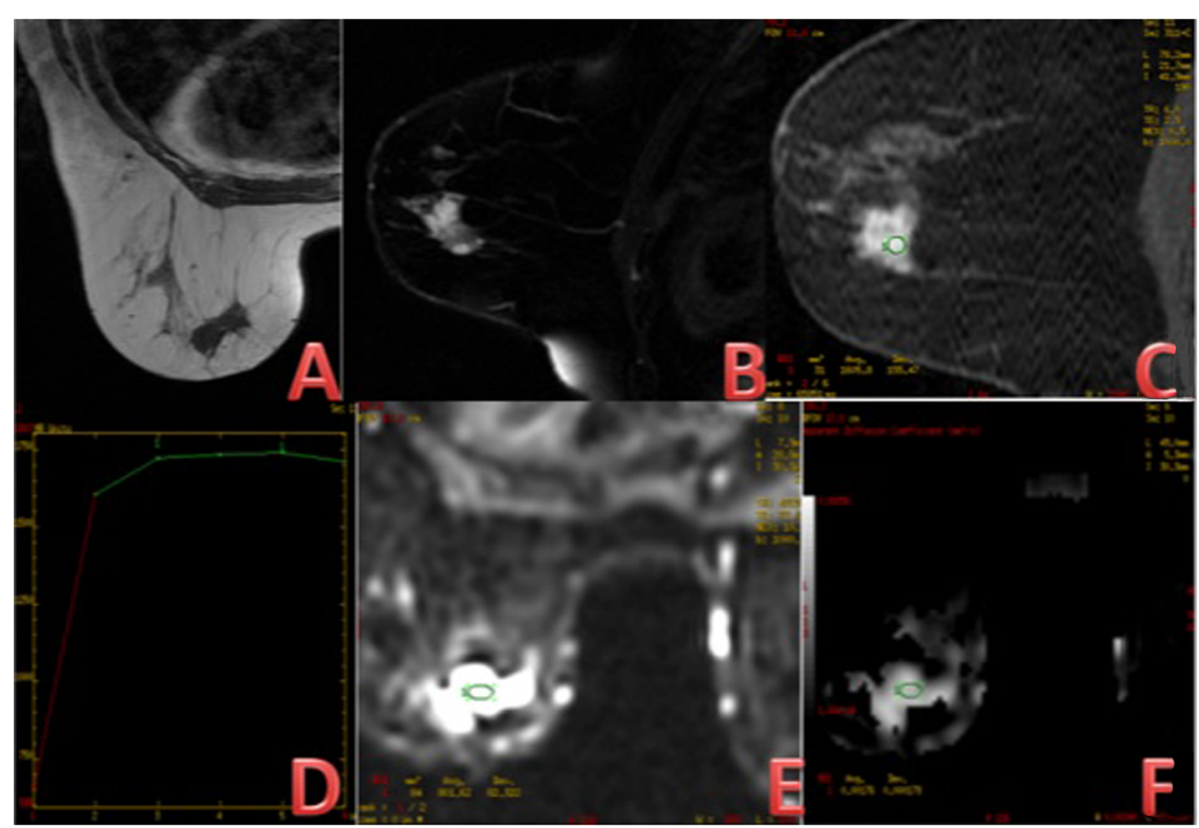

Fig. 2 56-year-old women, mucinous carcinoma, ER (+), PR (-) c-erb B2 (-), low Ki-67 index, and no axillary pathological lymph node detected on MRI. Breast density is classified as type c on T1Wl axial image (a). An irregular shaped lesion, measured $35 \mathrm{~mm}$ in diameter, is seen on sagittal fatsaturated T2WI (B) and sagittal fat-saturated, post-contrast T1WI (c). Kinetic curve of the lesion is type 2 and $t_{\text {peak }}$ value is $150 \mathrm{~s}$ (d). The lesion is hyperintense on DWI (e) and hypointense on ADC map (f) 


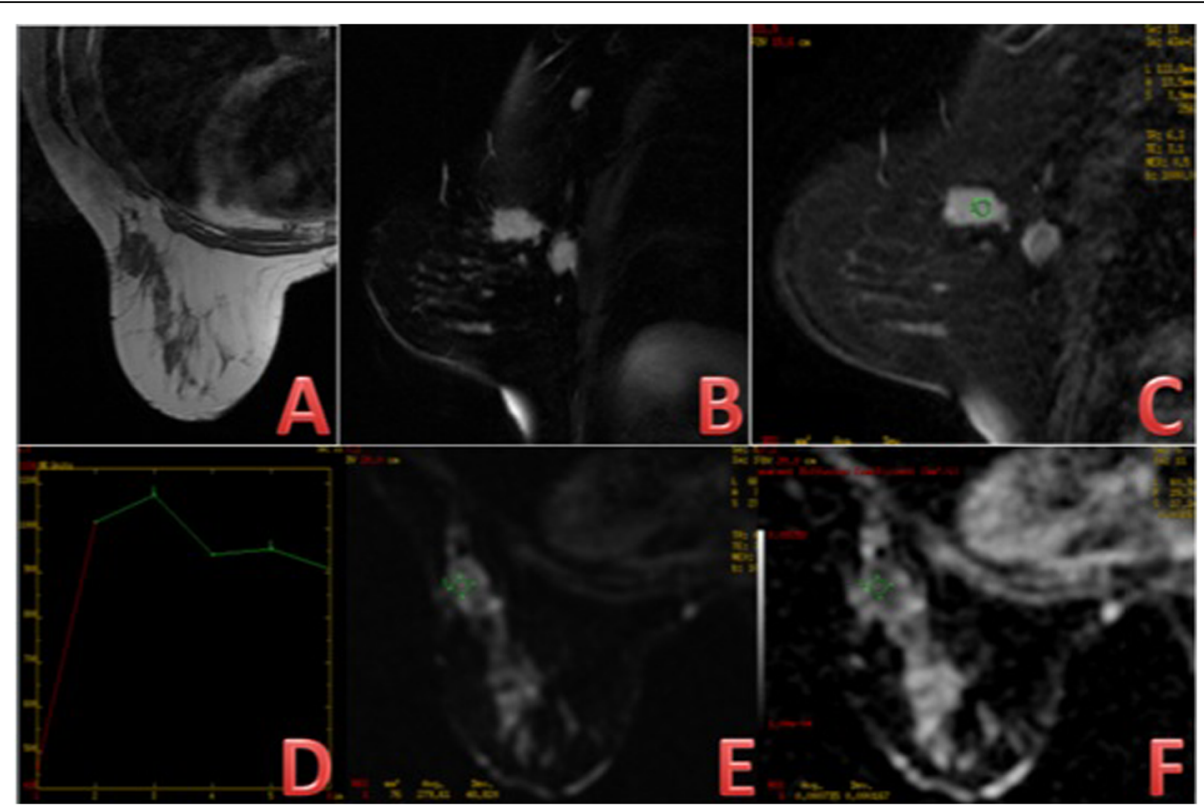

Fig. 3 54-year-old women, grade III neuroendocrine carcinoma, ER (-), PR (-) c-erb B2 (-), high Ki-67 index, and axillary pathological lymph node detected on MRI. Breast density is classified as type b on T1WI axial image (a). An irregular shaped lesion, measured $30 \mathrm{~mm}$ in diameter, is seen on sagittal fat-saturated T2WI (b) and sagittal fat-saturated, post-contrast T1WI (c). Kinetic curve of the lesion is type 3 and $t_{\text {peak }}$ value is $100 \mathrm{~s}$ (d). The lesion is hyperintense on DWI (e) and hypointense on ADC map (f)

$\mathrm{ADC}$ values were found to be related with ER-negative and PR-negative status and a higher Ki-67 index [33, 34]. On the other hand, a recent study found no correlation between ADC values and ER, PR, and Ki-67 expression [35]. We cannot define any relationship between ADC values and histopathological prognostic markers.

Current study has some limitations: first of all the retrospective nature of the study. Secondarily, ADC values cannot be found for some of the patients. Also, we cannot reach the results of every prognostic markers for all patients, seeing that we cannot evaluate every prognostic factor in all patients.

Using different MRI devices and protocols cause different results about kinetic curves. This might be the cause of contradictory results between our study and literature, and among previous studies. Also there is not a standardized $b$ value for ADC evaluation; these cause difficulties in comparing results of different studies. Twelve of 59 lesions of this study are smaller than $1 \mathrm{~cm}$ in diameter. For these kinds of lesions, 3-T MRI is better for evaluation. But our images were created by a $1.5-\mathrm{T}$ MRI device. Insufficiency in patient number is another limitation. Larger and more homogenous populations may provide clearer results.

\section{Conclusions}

Current study offers extensive information about the relationship between prognostic markers and MRI characteristics of malign breast lesions. A mild background enhancement, an intermediate signal intensity on T2WI, a high tpeak value, and absence of pathological axillary lymph node are found to be related with good prognostic factors. An irregular contour, a huge diameter, having a type III kinetic curve, a high slopei value, and presence of pathological axillary lymph node are found to be related with poor prognostic factors. ADC values are not related with prognostic factors. MRI can be used to predict prognostic factors in breast cancer cases.

\section{Abbreviations}

MRI: Magnetic resonance imaging; DWI: Diffusion-weighted imaging:

US: Ultrasonography; STIR: Spin echo short-tau inversion; EPI: Echo planar imaging; CER: Contrast enhancement ratio; IDC: Invasive ductal carcinoma; TC: Tubular carcinoma; DCIS: Ductal carcinoma in situ; ER: Estrogen receptor; PR: Progesterone receptor

\section{Acknowledgements}

Not applicable

\section{Authors' contributions}

KT and PSO analyzed and interpreted the patient data. SH and ENK helped in writing the manuscript. PK helped in literature search and editing the manuscript. PSO edited the last form of the manuscript. All authors read and approved the final manuscript.

\section{Funding}

No funding has been received for the study.

\section{Availability of data and materials}

The datasets used and/or analyzed during the current study are available from the corresponding author on reasonable request. 


\section{Ethics approval and consent to participate}

All procedures performed in studies involving human participants were in accordance with the ethical standards of the institutional and/or national research committee and with the 1964 Helsinki declaration and its later amendments or comparable ethical standards. The local institutional review board approved the study (Ankara Training and Research Hospital institutional review board reference number 0564-57857). Written informed consent was taken from all of the patients or from their parents if they were under the age of 18 .

\section{Consent for publication}

Informed consent was acquired from all participants.

\section{Competing interests}

The authors declare that they have no competing interests.

\section{Author details}

'Department of Radiology, Ankara Training and Research Hospital, Ankara, Turkey. ${ }^{2}$ Department of Pathology, Ankara Training and Research Hospital, Ankara, Turkey.

Received: 20 May 2020 Accepted: 22 July 2020

Published online: 10 August 2020

\section{References}

1. Leung JW (2005) Screening mammography reduces morbidity of breast cancer treatment. AJR Am J Roentgenol 184(5):1508-1509

2. Kolb TM, Lichy J, Newhouse JH (2002) Comparison of the performance of screening mammography, physical examination, and breast US and evaluation of factors that influence them: an analysis of 27,825 patient evaluations. Radiology. 225(1):165-175

3. Segel MC, Paulus DD, Hortobagyi GN (1988) Advanced primary breast cancer: assessment at mammography of response to induction chemotherapy. Radiology. 169(1):49-54

4. Rosen PP, Lesser ML, Arroyo CD, Cranor M, Borgen P, Norton L (1995) Immunohistochemical detection of HER2/neu in patients with axillary lymph node negative breast carcinoma. A study of epidemiologic risk factors, histologic features, and prognosis. Cancer. 75(6):1320-1326

5. Kuhl CK, Mielcareck P, Klaschik S, Leutner C, Wardelmann E, Gieseke J et al (1999) Dynamic breast MR imaging: are signal intensity time course data useful for differential diagnosis of enhancing lesions? Radiology. 211(1):101-110

6. Szabo BK, Aspelin P, Kristoffersen Wiberg M, Tot T, Bone B (2003) Invasive breast cancer: correlation of dynamic MR features with prognostic factors. Eur Radiol 13(11):2425-2435

7. Rakha EA, Putti TC, Abd El-Rehim DM, Paish C, Green AR, Powe DG et al (2006) Morphological and immunophenotypic analysis of breast carcinomas with basal and myoepithelial differentiation. J Pathol 208(4):495-506

8. Dilorenzo G, Telegrafo M, La Forgia D, Stabile lanora AA, Moschetta M (2019) Breast MRI background parenchymal enhancement as an imaging bridge to molecular cancer sub-type. Eur J Radiol 113:148-152

9. Kuhl CK, Schmutzler RK, Leutner CC, Kempe A, Wardelmann E, Hocke A et al (2000) Breast MR imaging screening in 192 women proved or suspected to be carriers of a breast cancer susceptibility gene: preliminary results. Radiology. 215(1):267-279

10. Nunes LW, Schnall MD, Orel SG (2001) Update of breast MR imaging architectural interpretation model. Radiology. 219(2):484-494

11. Kousi E, O'Flynn EAM, Borri M, Morgan VA, de Souza NM, Schmidt MA (2018) Pre-treatment functional MRI of breast cancer: T2* evaluation at $3 \mathrm{~T}$ and relationship to dynamic contrast-enhanced and diffusion-weighted imaging. Magn Reson Imaging 52:53-61

12. Lee SH, Cho N, Kim SJ, Cha JH, Cho KS, Ko ES et al (2008) Correlation between high resolution dynamic MR features and prognostic factors in breast cancer. Korean J Radiol 9(1):10-18

13. Kawashima H, Miyati T, Ohno N, Ohno M, Inokuchi M, Ikeda H et al (2017) Differentiation between luminal-A and luminal-B breast cancer using intravoxel incoherent motion and dynamic contrast-enhanced magnetic resonance imaging. Acad Radiol 24(12):1575-1581

14. Mussurakis S, Buckley DL, Horsman A (1997) Dynamic MR imaging of invasive breast cancer: correlation with tumour grade and other histological factors. Br J Radiol 70(833):446-451
15. Stomper PC, Herman S, Klippenstein DL, Winston JS, Budnick RM, Stewart CC (1996) Invasive breast carcinoma: analysis of dynamic magnetic resonance imaging enhancement features and cell proliferative activity determined by DNA S-phase percentage. Cancer. 77(9):1844-1849

16. Baltzer PA, Vag T, Dietzel M, Beger S, Freiberg C, Gajda M et al (2010) Computer-aided interpretation of dynamic magnetic resonance imaging reflects histopathology of invasive breast cancer. Eur Radiol 20(7):1563-1571

17. Ponzone R, Montemurro F, Maggiorotto F, Robba C, Gregori D, Jacomuzzi ME et al (2006) Clinical outcome of adjuvant endocrine treatment according to PR and HER-2 status in early breast cancer. Ann Oncol 17(11):1631-1636

18. Bone B, Aspelin P, Isberg B, Perbeck L, Veress B (1995) Contrast-enhanced MR imaging of the breast in patients with breast implants after cancer surgery. Acta Radiol 36(2):111-116

19. Mussurakis S, Buckley DL, Horsman A (1997) Dynamic MRI of invasive breast cancer: assessment of three region-of-interest analysis methods. J Comput Assist Tomogr 21(3):431-438

20. Fischer U, Kopka L, Brinck U, Korabiowska M, Schauer A, Grabbe E (1997) Prognostic value of contrast-enhanced MR mammography in patients with breast cancer. Eur Radiol 7(7):1002-1005

21. Stomper PC, Herman S, Klippenstein DL, Winston JS, Edge SB, Arredondo MA et al (1995) Suspect breast lesions: findings at dynamic gadoliniumenhanced MR imaging correlated with mammographic and pathologic features. Radiology. 197(2):387-395

22. Agrawal G, Chen JH, Baek HM, Hsiang D, Mehta RS, Nalcioglu O et al (2007) MRI features of breast cancer: a correlation study with HER-2 receptor. Ann Oncol 18(11):1903-1904

23. Tuncbilek N, Karakas HM, Okten $\mathrm{OO}$ (2005) Dynamic magnetic resonance imaging in determining histopathological prognostic factors of invasive breast cancers. Eur J Radiol 53(2):199-205

24. Fan M, Cheng H, Zhang P, Gao X, Zhang J, Shao G et al (2018) DCE-MRl texture analysis with tumor subregion partitioning for predicting Ki-67 status of estrogen receptor-positive breast cancers. J Magn Reson Imaging 48(1):237-247

25. Caiazzo C, Di Micco R, Esposito E, Sollazzo V, Cervotti M, Varelli C et al (2018) The role of MRI in predicting Ki-67 in breast cancer: preliminary results from a prospective study. Tumori. 104(6):438-443

26. Tresserra F, Rodriquez I, Garcia-Yuste M, Grases PJ, Ara C, Fabregas R (2007) Tumor size and lymph node status in multifocal breast cancer. Breast J 13(1):68-71

27. Wrba F, Chott A, Reiner A, Reiner G, Markis-Ritzinger E, Holzner JH (1989) Ki67 immunoreactivity in breast carcinomas in relation to transferrin receptor expression, estrogen receptor status and morphological criteria. An immunohistochemical study. Oncology. 46(4):255-259

28. Thomas GA, Leonard RC (2009) How age affects the biology of breast cancer. Clin Oncol 21(2):81-85

29. Parise CA, Bauer KR, Caggiano V (2010) Variation in breast cancer subtypes with age and race/ethnicity. Crit Rev Oncol Hematol 76(1):44-52

30. Albain KS, Allred DC, Clark GM (1994) Breast cancer outcome and predictors of outcome: are there age differentials? I Natl Cancer Inst Monogr 16:35-42

31. Razek AA, Gaballa G, Denewer A, Nada N (2010) Invasive ductal carcinoma: correlation of apparent diffusion coefficient value with pathological prognostic factors. NMR Biomed 23(6):619-623

32. Kamitani T, Matsuo Y, Yabuuchi H, Fujita N, Nagao M, Jinnouchi M et al (2013) Correlations between apparent diffusion coefficient values and prognostic factors of breast cancer. Magnetic resonance in medical sciences :MRMS : an official journal of Japan Society of Magnetic Resonance in Medicine 12(3):193-199

33. Ren C, Zou Y, Zhang X, Li K (2019) Diagnostic value of diffusion-weighted imaging-derived apparent diffusion coefficient and its association with histological prognostic factors in breast cancer. Oncol Lett 18(3):3295-3303

34. Kim EJ, Kim SH, Park GE, Kang BJ, Song BJ, Kim YJ et al (2015) Histogram analysis of apparent diffusion coefficient at $3.0 \mathrm{t}$ : correlation with prognostic factors and subtypes of invasive ductal carcinoma. J Magn Reson Imaging 42(6):1666-1678

35. Yuan C, Jin F, Guo X, Zhao S, Li W, Guo H (2019) Correlation analysis of breast cancer DWI combined with DCE-MRI imaging features with molecular subtypes and prognostic factors. J Med Syst 43(4):83

\section{Publisher's Note}

Springer Nature remains neutral with regard to jurisdictional claims in published maps and institutional affiliations. 